\title{
Dynamic Rule Encryption for Mobile Payment
}

\author{
Emir Husni \\ School of Electrical Engineering \& Informatics, Institut Teknologi Bandung, Jawa Barat, Indonesia \\ Correspondence should be addressed to Emir Husni; ehusni@lskk.ee.itb.ac.id \\ Received 11 October 2016; Revised 8 December 2016; Accepted 18 December 2016; Published 26 January 2017 \\ Academic Editor: Ángel Martín Del Rey \\ Copyright (c) 2017 Emir Husni. This is an open access article distributed under the Creative Commons Attribution License, which \\ permits unrestricted use, distribution, and reproduction in any medium, provided the original work is properly cited.

\begin{abstract}
The trend of financial transactions by using a mobile phone or mobile payment increases. By using the mobile payment service, users can save money on mobile phone (handset) and separate from the pulse. For protecting users, mobile payment service providers must complete the mobile payment service with the transaction security. One way to provide transaction security is to utilize a secure mobile payment application. This research provides a safety feature used for an Android-based mobile payment application. This security feature is making encryption rules dynamically named Dynamic Rule Encryption (DRE). DRE has the ability to protect data by means of encrypting data with dynamic rules, and DRE also has a token function for an authentication. DRE token raised with dynamic time-based rules. Here, the time is used as a reference with the order of the day in the year (day of the year). The processes of the DRE's encryption, decryption, and the DRE's functionality as the token are discussed in this paper. Here, the Hamming distance metric is employed for having maximum differences between plaintext and ciphertext.
\end{abstract}

\section{Introduction}

The current trend is numbers of mobile phones decreased slowly while smartphones are growing rapidly. In addition, the computing resources in smartphone improving yearly with more applications can be integrated into it. Due to applications, smartphone is an electronic device that is most commonly used. A sort of smartphone apps has been made by the developers in Indonesia, for example: GO-JEK (online taxi motorbike) has successfully run using smartphone [1], mobile-based academic information system [2], shopping using smartphone [3], mobile payment $[4,5]$, and mobile service commerce [6].

Trends in financial transactions using a mobile phone or mobile payment increase. The numbers of operators providing such services also increased. By employing the mobile payment service, customers can save money on mobile phone (handset) and separate from the main content. Thus, the mobile phone serves as an electronic wallet (mobile wallet).

Currently, most of Android smartphones support Near Field Communication (NFC). The NFC has been implemented in shopping [3] and a mobile payment application [4, 5].

The mobile payment service provider should complete mobile payment service with a good transaction security. One path to provide transaction security is to utilize secure mobile payment applications. Mobile payment applications should have good security because the application will contain sensitive data, including account, password, PIN, e-money, and transaction data. This research offers a security feature used for mobile payment applications on the Android operating system. This security feature is a dynamic encryption rule named Dynamic Rule Encryption (DRE). DRE has the power to protect information by encrypting data with dynamic rules, and DRE also has a function as a token for authentication. Token raised DRE using dynamic rules or change by time. The reference period used is on the order of the day in the year (day of the year). Many existing rules on DRE are limited to certain time. This causes the device to be connected to the server to update the rules DRE. By connecting to the server, the server can validate the existing data on the mobile payment application, so that if there are invalid data, the data is detected. In addition, the DRE token contains information about the device, time, mobile payment user's account, and other information used for authentication.

\section{Literature Survey}

2.1. Encryption. One element that should be owned by the sensitive data is data security. Sensitive data should have 


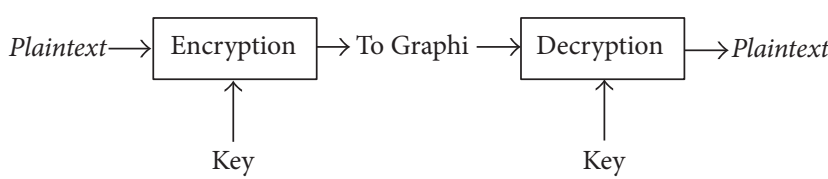

FIGURE 1: Encryption process.

dependable data security; both when it is stored on storage media or when transferred to another location via any channel, for example, via the Internet. Data security can be constructed in various ways, one of which is by using encryption of the data to be stored or transferred.

Encryption can also be named a secret password (cipher). But the actual encryption is only one part of a secret password. Secret password consists of encryption and decryption. Encryption is the procedure of encoding the information so that the information cannot be read by normal means. Encryption will change the data on the information that will be hard to read and time consuming if the encryption result data decoded without using any special rules or codes.

Encryption involves secret data called key. The keys are also employed in the decryption process. Without using a key, the information will not be encrypted or decrypted. The information input into encryption called plaintext and encrypted data is called ciphertext. The ciphertext message contains all the information of the plaintext message but not in format that is obtained and read normally. Decryption process is required to restore the plaintext to ciphertext. The block diagram of encryption process is shown in Figure 1.

2.1.1. Encryption Type. By the type of the key, encryption is divided into two types, namely:

(1) Symmetric encryption, and

(2) Asymmetric encryption.

Symmetric encryption is encrypted using the same key for encryption and decryption, while the asymmetric encryption is encrypted using different keys for encryption and decryption, namely, a public key and a private key. Symmetric encryption includes AES, DES, and Triple DES. Asymmetric encryption includes RSA.

Looking at ways of processing the data, there are two types of encryption, namely:

(1) The stream cipher, and

(2) Block cipher.

Stream ciphers are used in the symmetric encryption, while the block ciphers are used in symmetric and asymmetric encryption. The difference between stream ciphers and block cipher is block ciphers process data in data blocks, while stream ciphers process data one bit or one byte at a time.

2.1.2. AES (Advanced Encryption Standard). AES is a type of encryption using symmetric keys and a block cipher [7]. AES consists of three types, namely, AES-128, AES-192, and AES256. Each has a size of 128-bit block cipher with a key size

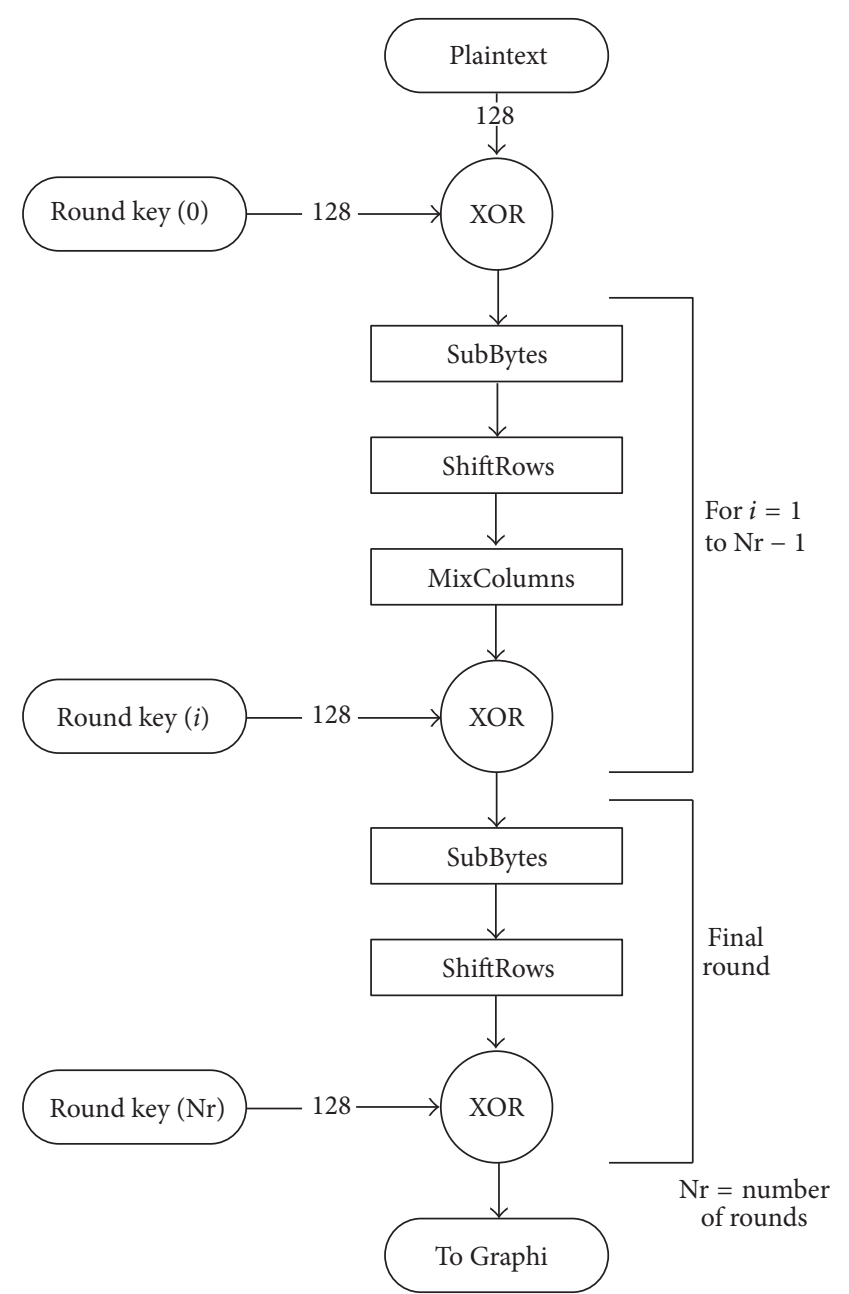

FIGURE 2: AES encryption process.

of 128,192 , and 256 bits. AES has been analyzed extensively and is widely used throughout the world. From the size of the blocks, AES works on a $4 \times 4$ matrix in which each cell of the matrix consists of a 1 byte ( 8 bits); then the size of a block is 16 bytes. At AES, there are 4 main stages to process the incoming data, namely, stage SubBytes, ShiftRow, MixColumn, and add round key. Figure 2 illustrates the AES encryption process.

2.1.3. DES (Data Encryption Standard). DES is also a type of encryption using symmetric keys and a block cipher [8]. DES has a 64-bit block size. DES encrypts 64 bits of plaintext into 64 bits ciphertext using a 56-bit subkey generated from the master key of 64 bits. In the process of encryption and decryption, DES uses Feistel network. The DES encryption process is illustrated in Figure 3.

2.2. Token. The token is software or hardware used for authentication. Tokens are used to prove identity electronically as well as being used as electronic key to access something. Tokens can be used instead of the password security feature or used in conjunction with a password [9]. 


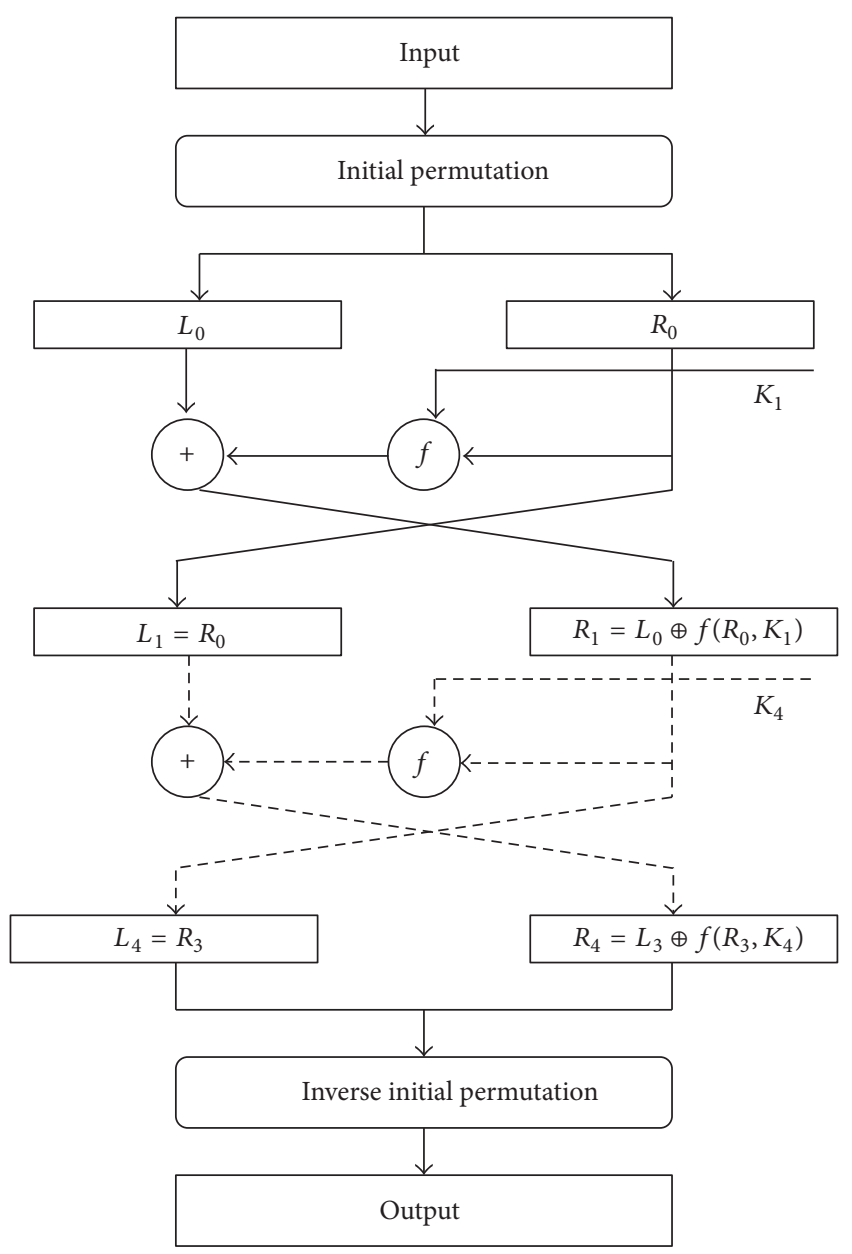

FIGURE 3: DES encryption process.

2.2.1. Forms of Tokens. Tokens may take software or hardware. At hardware token, there is a chip. The chip has a function that varies from simple to complex. Tokens must have a certification indicating that the token meets various security standards, including meeting government security standards, industry security standards, testing standards, and other cryptographic standards. Here are some forms of token.

(1) Disconnected Token. Disconnected Token has no connection to the access point device or computer. This token has display for displaying data or authentication serial numbers raised. That serial number will be filled in the field token (passcode) program interface, computer, or other device to be accessed. This token is one type of tokens most widely used.

(2) Connected Token. Connected Token is a physical token which must be connected to the device or the computer to be accessed. This token will automatically authenticate when connected to any device or computer. The most common types of physical tokens are smart cards and USB tokens.

(3) Contactless Token. Contactless token is almost the same as the Connected Token. Contactless token is connected to a device or computer access point. Contactless tokens are

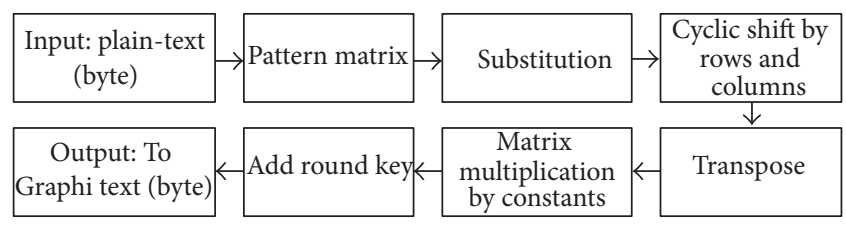

FIGURE 4: Stages of algorithm.

just as Connected Token, but, contactless tokens are not physically connected. One way contactless tokens connected to any device or computer is by using RFID.

(4) Bluetooth Tokens. Bluetooth token is often combined with a USB token; that Bluetooth token can work either in a state connected or not connected physically to the device or a computer access point. Authentication using this token works when it is less than ten meters from the device or computer. When a Bluetooth mode does not work, then the token must be connected via USB. The advantage of using this token is the ability to sign-off from a certain distance (less than ten meters).

(5) GSM Cellular Phone Token. GSM Cellular Phone token is GSM enabled mobile phone as a token. How to use this token is to install a token program (Java) on your phone. Another way is to use SMS, phone calls, and over Internet protocol (HTTP/HTTPS). There is a token called a mobile device token. Mobile device tokens are almost the same as GSM Cellular Phone tokens.

\section{Dynamic Rule Encryption}

Dynamic Rule Encryption (DRE) is a symmetric block cipher with a symmetrical key [10]. DRE cipher block size is 512 bits, while key length is 128 bits. On DRE there are 6 steps done in plaintext, namely:

(1) Pattern matrix formation [11]

(2) Substitution [12]

(3) Cyclic shift by rows and columns [13]

(4) Transpose [12]

(5) The matrix multiplication by constants [12], and

(6) Add round key [12]

Substitution, cyclic shift, and transpose phases are functioned as confusion and diffusion. Confusion serves to establish the correlation between the ciphertext and the key when it is misplaced or not visible. Confusion is a nonlinear function [12]. Diffusion makes a correlation between the plaintext and ciphertext missing or not visible [12]. By using diffusion, the code will be difficult to resolve using statistical methods.

In DRE, the sequence of steps may be changing over time. Figure 4 is one example of these stages. At other times, the sequence of steps turns out to be as in Figure 5.

Step changes are explained in Figures 4 and 5 just as one example. DRE has a sequence pattern change. In addition, the 


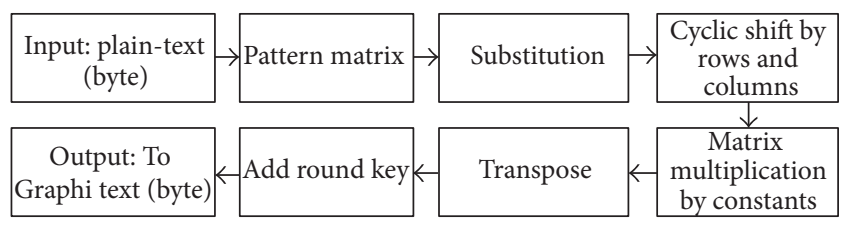

FIGURE 5: Stages of algorithm with different pattern.

DRE has the elements or variables that may change on time, that is:

(1) Placement pattern input of the matrix

(2) The substituted matrix elements

(3) The rows and columns shifted and amounts of shift operation

(4) The constant matrix, and

(5) The master key to add a round key stage

3.1. Encryption Process. Here is an explanation of the encryption process.

(1) Matrix Pattern Formation. At this level, the DRE's input will be separated into several parts and these parts will be combined with a pattern. The placement pattern of the matrix input is changing over time. Figures 6 and 7 provide an example of the matrix pattern change. Figure 6 shows one matrix pattern, in which the matrix is consisting of six parts with different colors. When the time changes, the matrix in Figure 6 will be turned into the matrix in Figure 7. As we can see that the matrix pattern is changed on time.

(2) Substitution. At this stage, byte elements in a matrix are substituted with other values on look-up table. The number of elements in the matrix to be substituted is as much as 48 bytes elements. A byte substitution matrix element is determined by the bit pattern. Suppose bits in the byte matrix element are b7, b6, b5, b4, b3, b2, b1, and b0, where b0 = LSB and b7 = MSB. Couple substitutions are determined by changing the sequence of bits in a byte following the pattern of bits b7, b2, b3, b4, b5, b6, b1, and b0. Substitution of byte matrix elements is carried per nibble of the byte; that is, b7, b3, b5, and b1 are called nibble 1 and b2, b4, b6, and b0 are called nibble 2. Substitution is done by seeing the look-up table. Table 1 is a look-up table to nibble 1 (top) and to nibble 2 (bottom). Next in Table 2 there is an example of the substitution matrix element.

(3) Cyclic Shift by Rows and Columns. At this stage, byte elements in the matrix will be shifted in rows or/and columns. Locations of rows and columns shifted and the amount of every shift can vary based on time. This shift can also be called a rotation. Here is an example of the cyclic shift for a $4 \times 4$ matrix. Equation (1) is an example of the cyclic in rows with the amount of shift shown in Table 3, while (2) is an example
TABLE 1: Substitution look-up table.

\begin{tabular}{|c|c|c|}
\hline & Input & Output \\
\hline \multirow{16}{*}{ Nibble 1} & 0000 & 0111 \\
\hline & 0001 & 1110 \\
\hline & 0010 & 0100 \\
\hline & 0011 & 1101 \\
\hline & 0100 & 0001 \\
\hline & 0101 & 0010 \\
\hline & 0110 & 1111 \\
\hline & 0111 & 1011 \\
\hline & 1000 & 0011 \\
\hline & 1001 & 1010 \\
\hline & 1010 & 1000 \\
\hline & 1011 & 0110 \\
\hline & 1100 & 0101 \\
\hline & 1101 & 1100 \\
\hline & 1110 & 1001 \\
\hline & 1111 & 0000 \\
\hline \multirow{16}{*}{ Nibble 2} & 0000 & 1101 \\
\hline & 0001 & 1111 \\
\hline & 0010 & 1100 \\
\hline & 0011 & 1000 \\
\hline & 0100 & 0010 \\
\hline & 0101 & 0100 \\
\hline & 0110 & 1001 \\
\hline & 0111 & 0001 \\
\hline & 1000 & 0111 \\
\hline & 1001 & 0101 \\
\hline & 1010 & 1011 \\
\hline & 1011 & 0011 \\
\hline & 1100 & 1110 \\
\hline & 1101 & 1010 \\
\hline & 1110 & 0000 \\
\hline & 1111 & 0110 \\
\hline
\end{tabular}

of the cyclic in columns with the amount of shift shown in Table 4.

The Example of Row Cyclic Sliding

$\left[\begin{array}{llll}01100011 & 10100101 & 01000100 & 00001000 \\ 00000000 & 01100100 & 00110100 & 10000100 \\ 00101011 & 01011000 & 00100001 & 01011111 \\ 00110000 & 00000101 & 11111100 & 00010011\end{array}\right]$

$\downarrow$ 
TABLE 2: Example of matrix element substitution.

\begin{tabular}{lccr}
\hline Input & Pattern & & \\
& Nibble 2 (b7 b3 b5 b1) & Nibble 1 (b6 b2 b4 b0) & Output \\
\hline 10001001 & 1100 & 0001 & 11101110 \\
01100010 & 0011 & 1000 & 10000011 \\
01100101 & 0010 & 1101 & 11001100 \\
10000011 & 1001 & 0001 & 01011110 \\
\hline
\end{tabular}

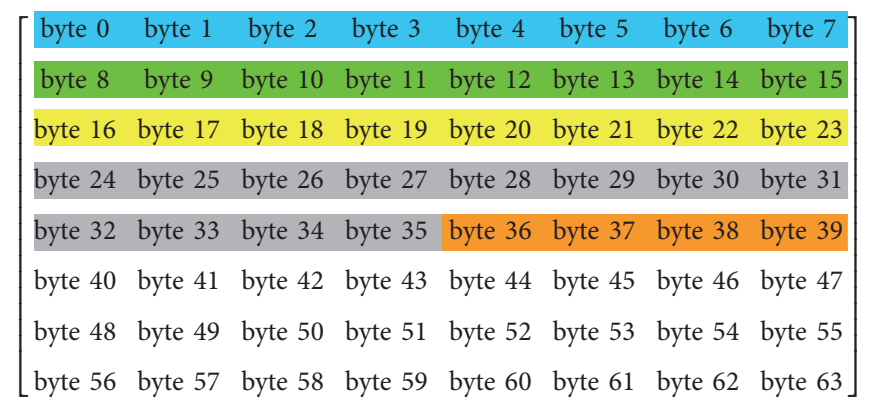

FIGURE 6: Matrix's parts 1. Explanation: blue $=$ SIM, green $=$ IMEI, yellow $=$ time stamp, gray $=$ account number, orange $=$ big transaction, and white $=$ salt.

$\left[\begin{array}{llllllll}\text { byte } 0 & \text { byte } 1 & \text { byte } 2 & \text { byte } 3 & \text { byte } 4 & \text { byte } 5 & \text { byte } 6 & \text { byte } 7 \\ \text { byte } 8 & \text { byte } 9 & \text { byte } 10 & \text { byte } 11 & \text { byte } 12 & \text { byte } 13 & \text { byte } 14 & \text { byte } 15 \\ \text { byte } 16 & \text { byte } 17 & \text { byte } 18 & \text { byte } 19 & \text { byte } 20 & \text { byte } 21 & \text { byte } 22 & \text { byte } 23 \\ \text { byte } 24 & \text { byte } 25 & \text { byte } 26 & \text { byte } 27 & \text { byte } 28 & \text { byte } 29 & \text { byte } 30 & \text { byte } 31 \\ \text { byte } 32 & \text { byte } 33 & \text { byte } 34 & \text { byte } 35 & \text { byte } 36 & \text { byte } 37 & \text { byte } 38 & \text { byte } 39 \\ \text { byte } 40 & \text { byte } 41 & \text { byte } 42 & \text { byte } 43 & \text { byte } 44 & \text { byte } 45 & \text { byte } 46 & \text { byte } 47 \\ \text { byte } 48 & \text { byte } 49 & \text { byte } 50 & \text { byte } 51 & \text { byte } 52 & \text { byte } 53 & \text { byte } 54 & \text { byte } 55 \\ \text { byte } 56 & \text { byte } 57 & \text { byte } 58 & \text { byte } 59 & \text { byte } 60 & \text { byte } 61 & \text { byte } 62 & \text { byte } 63\end{array}\right]$

FIGURE 7: Matrix's parts 2. Explanation: blue $=$ SIM, green $=$ IMEI, yellow $=$ time stamp, gray $=$ account number, orange $=$ big transaction, and white $=$ salt.

TABLE 3: The example of row cyclic shift.

\begin{tabular}{lllll}
\hline Row & 1 & 2 & 3 & 4 \\
\hline Number of cyclic shift to left & 0 & 1 & 2 & 3 \\
\hline
\end{tabular}

\section{The Example of Column Cyclic Sliding}

$$
\left[\begin{array}{llll}
01100011 & 10100101 & 01000100 & 00001000 \\
01100100 & 00110100 & 10000100 & 00000000 \\
00100001 & 01011111 & 00101011 & 01011000 \\
00010011 & 00110000 & 00000101 & 11111100
\end{array}\right]
$$

$$
\downarrow
$$

$$
\left[\begin{array}{llll}
01100011 & 00110100 & 00101011 & 11111100 \\
01100100 & 01011111 & 00000101 & 00001000 \\
00100001 & 00110000 & 01000100 & 00000000 \\
00010011 & 10100101 & 10000100 & 01011000
\end{array}\right]
$$

(4) Transpose. This stage is the matrix transpose operation. The transpose of a matrix is a new matrix whose rows are the columns of the original. Equation (3) is an example of the matrix transpose operation on a $4 \times 4$ matrix.

The Example of Transpose on a $4 \times 4$ Matrix

$$
\left[\begin{array}{cccc}
\text { byte } 0 & \text { byte } 1 & \text { byte } 2 & \text { byte } 3 \\
\text { byte } 4 & \text { byte } 5 & \text { byte } 6 & \text { byte } 7 \\
\text { byte } 8 & \text { byte } 9 & \text { byte } 10 & \text { byte } 11 \\
\text { byte } 12 & \text { byte } 13 & \text { byte } 14 & \text { byte } 15
\end{array}\right]
$$

$$
\downarrow
$$

$\left[\begin{array}{llll}\text { byte } 0 & \text { byte } 4 & \text { byte } 8 & \text { byte } 12 \\ \text { byte } 1 & \text { byte } 5 & \text { byte } 9 & \text { byte } 13 \\ \text { byte } 2 & \text { byte } 6 & \text { byte } 10 & \text { byte } 14 \\ \text { byte } 3 & \text { byte } 7 & \text { byte } 11 & \text { byte } 15\end{array}\right]$

(5) Matrix Multiplication by Constants. In this stage, the input matrix is multiplied by a constant element matrix. The matrix 
TABLE 4: The example of column cyclic shift.

\begin{tabular}{lllll}
\hline Column & 1 & 2 & 3 & 4 \\
\hline Number of cyclic shift up & 0 & 1 & 2 & 3 \\
\hline
\end{tabular}

elements are multiplied in nibble format. Matrix's constants as multipliers can vary based on the time. Multiplication of matrices with a constant matrix is done in Galois field $\mathrm{GF}\left(2^{4}\right)$. Equation (4) is an example of one of the constants' matrix in $\mathrm{GF}\left(2^{4}\right)$.

The Example of a Constant Matrix in $G F\left(2^{4}\right)$

$$
\left[\begin{array}{llllllll}
3 & 4 & 5 & 6 & 2 & 2 & 2 & 2 \\
2 & 3 & 4 & 5 & 6 & 2 & 2 & 2 \\
2 & 2 & 3 & 4 & 5 & 6 & 2 & 2 \\
2 & 2 & 2 & 3 & 4 & 5 & 6 & 2 \\
2 & 2 & 2 & 2 & 3 & 4 & 5 & 6 \\
6 & 2 & 2 & 2 & 2 & 3 & 4 & 5 \\
5 & 6 & 2 & 2 & 2 & 2 & 3 & 4 \\
4 & 5 & 6 & 2 & 2 & 2 & 2 & 3
\end{array}\right]
$$

(6) Add Round Key. At add round key operation, a matrix element is combined with a block of the round key using bitwise xor. Round using 128-bits master key and master key will be expanded to produce a 512-bits subkey. When building a subkey, this process involves auxiliary matrix $M$ that contains the master key and iteration with a modulus of 255. Equation (5) is a matrix $M$ generated from the master keys 4 C, 69, 66, 65, 27, 73, 20, 62, 65, 61, 75, 74, 69, 66, 75, and $6 \mathrm{C}$,where Figure 8 is an algorithm to expand the master key of 128 bits into the 512-bit subkey.

\section{The M Matrix}

$$
\left[\begin{array}{ccccc}
76 & 77 & 78 & \ldots & \ldots \\
105 & 106 & 107 & \ldots & \ldots \\
102 & 103 & 104 & \ldots & \ldots \\
101 & 102 & 103 & \ldots & \ldots \\
39 & 40 & 41 & \ldots & \ldots \\
115 & 116 & 117 & \ldots & \ldots \\
32 & 33 & 34 & \ldots & \ldots \\
98 & 99 & 100 & \ldots & \ldots \\
101 & 102 & 103 & \ldots & \ldots \\
97 & 98 & 99 & \ldots & \ldots \\
117 & 118 & 119 & \ldots & \ldots \\
116 & 117 & 118 & \ldots & \ldots \\
105 & 106 & 107 & \ldots & \ldots \\
102 & 103 & 104 & \ldots & \ldots \\
118 & 119 & 120 & \ldots & \ldots \\
108 & 109 & 110 & \ldots & \ldots
\end{array}\right]
$$

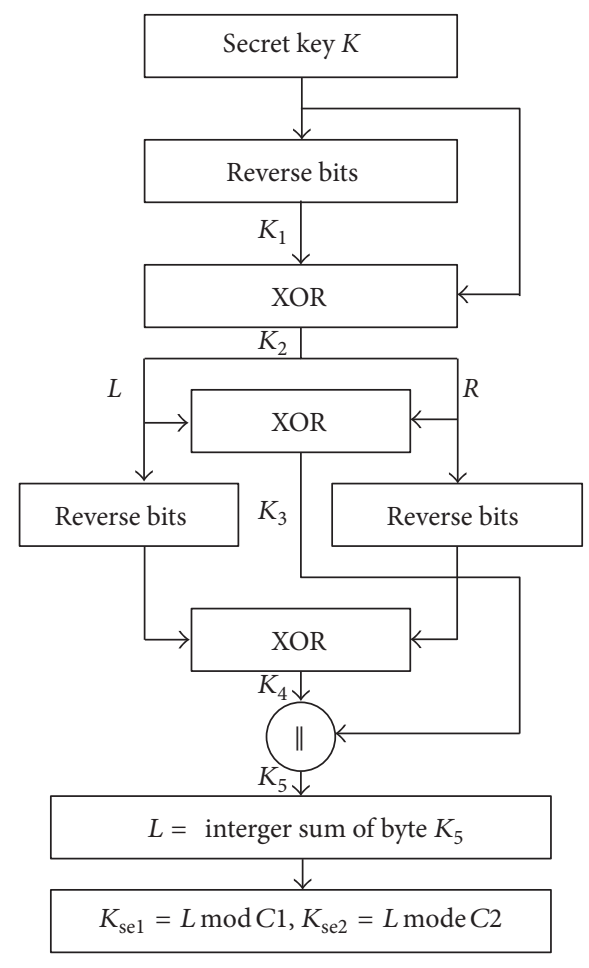

FIGURE 8: Master key expansion algorithm.

The master key expansion procedure is as follows.

(1) Master key or hidden key, $K$, is transposed to gain $K_{1}$.

(2) $K_{2}$ is obtained by performing xor operation between $K$ and $K_{1}$.

(3) With the column, $K_{2}$ is divided into two, namely, the right and the left. Both parts are made xor operation to gain $K_{3}$.

(4) The right side and the left side of $K_{2}$ are transposed. Both parts are then transposed. The results of operations are made xor operation to gain $K_{4}$.

(5) $K_{3}$ and $K_{4}$ are concatenated (united) to get $K_{5}$.

(6) The byte integer value of $K_{5}$ is summed to obtain $L$.

(7) $K_{\text {sel }}$ is calculated by the formula $K_{\text {se1 }}=L \bmod C 1$, with $C 1=23$.

(8) $K_{\mathrm{se} 2}$ is calculated by the formula $K_{\mathrm{se} 2}=L \bmod C 2$, with $C 2=15$.

(9) Then, the subkey $K_{\mathrm{s} 2}$ is obtained from the decrease of auxiliary matrix $M$ as follows.

(i) $K_{\mathrm{s} 1}[$ row $]$ column $]=M[$ row $]\left[K_{\text {se1 }}+K_{\text {se2 }}+\right.$ column].

(ii) $K_{\mathrm{s} 2}$ [row $]$ [column] $=M[$ row $]\left[K_{\mathrm{s} 1}\right.$ [row $]$ [column]].

(10) While, subkey $K_{\mathrm{s} 1}$ is obtained from the decrease of auxiliary matrix $M$ as follows. 
(i) $K_{\mathrm{s} 1}[$ row $][$ column $]=M[$ row $]\left[K_{\mathrm{s} 2}[\right.$ row $]$ [column]].

(ii) $i$ th column of the matrix $K_{\mathrm{s} 1}$ rotated vertically downwards as far as $\left(\left((\operatorname{int}(K[i]) \bmod 12)+K_{\mathrm{sel}}\right)\right.$ $\bmod 15)$.

(iii) $i$ th column of the matrix $K_{\mathrm{s} 2}$ rotated vertically downwards as far as $\left(\left((\operatorname{int}(K[i]) \bmod 10)+K_{\text {se1 }}\right)\right.$ $\bmod 15)$.
In [14], the algorithm in Figure 8 can generate many changes on the subkey bits when little change occurs on the master key. $K_{\mathrm{s} 1}$ and $K_{\mathrm{s} 2}$ each subkey will generate 256 bits; then the total subkey generated is 512 pieces. Equation (6) is an example of some subkey generated from the master key $4 \mathrm{C}$ 6966652773206265617574696675 6C.

The Example of Some Subkey Produced by Master Key

$\begin{array}{llllllllllllllll}6 \mathrm{D} & 6 \mathrm{~A} & 49 & 40 & 6 \mathrm{D} & 52 & \mathrm{~F} 2 & 49 & 40 & 3 \mathrm{D} & 83 & 67 & 6 \mathrm{E} & 34 & 3 \mathrm{D} & 31 \\ 68 & 6 \mathrm{~F} & 35 & 3 \mathrm{E} & 32 & 6 \mathrm{E} & 6 \mathrm{~B} & 4 \mathrm{~A} & 41 & 6 \mathrm{E} & 53 & \mathrm{~F} 3 & 4 \mathrm{~A} & 41 & 3 \mathrm{E} & 84 \\ 3 \mathrm{~F} & 33 & 6 \mathrm{~F} & 6 \mathrm{C} & 4 \mathrm{~B} & 42 & 6 \mathrm{~F} & 54 & \mathrm{~F} 4 & 4 \mathrm{~B} & 42 & 3 \mathrm{~F} & 85 & 69 & 70 & 36 \\ 34 & 70 & 6 \mathrm{D} & 4 \mathrm{C} & 43 & 70 & 55 & \mathrm{~F} 5 & 4 \mathrm{C} & 43 & 40 & 86 & 6 \mathrm{~A} & 71 & 37 & 40 \\ 6 \mathrm{E} & 4 \mathrm{D} & 44 & 71 & 56 & \mathrm{~F} 6 & 4 \mathrm{D} & 44 & 41 & 87 & 6 \mathrm{~B} & 72 & 38 & 41 & 35 & 71 \\ 39 & 42 & 36 & 72 & 6 \mathrm{~F} & 4 \mathrm{E} & 45 & 72 & 57 & \mathrm{~F} 7 & 4 \mathrm{E} & 45 & 42 & 88 & 6 \mathrm{C} & 73 \\ 74 & 3 \mathrm{~A} & 43 & 37 & 73 & 70 & 4 \mathrm{~F} & 46 & 73 & 58 & \mathrm{~F} 8 & 4 \mathrm{~F} & 46 & 43 & 69 & 6 \mathrm{D} \\ 50 & 47 & 74 & 59 & \mathrm{~F} 9 & 50 & 47 & 44 & 8 \mathrm{~A} & 6 \mathrm{E} & 75 & 3 \mathrm{~B} & 44 & 38 & 74 & 71 \\ 39 & 75 & 72 & 51 & 48 & 75 & 5 \mathrm{~A} & \mathrm{FA} & 51 & 48 & 45 & 8 \mathrm{~B} & 6 \mathrm{~F} & 76 & 3 \mathrm{C} & 45 \\ 49 & 76 & 5 \mathrm{~B} & \mathrm{FB} & 52 & 49 & 46 & 8 \mathrm{C} & 70 & 77 & 3 \mathrm{D} & 46 & 3 \mathrm{~A} & 76 & 73 & 52\end{array}$

After the plaintext experiences the 6 steps above, the plaintext will change into the ciphertext.

\subsection{Process Description. Here is an explanation of the process} on the description.

(1) Inverse Substitution. This stage is the reverse of substitution. Inverse substitution is done using an inverse substitution table. The rules for determining the inverse substitution couple equally with the existing rules at the stage of encryption substitution. The byte substitution matrix element is done by looking at the inverse substitution look-up table as shown in Table 5.

(2) Inverse Cyclic Shift by Rows and Columns. This stage is the contrary of the cyclic shift by row and column in the encoding process. The magnitude and direction of the shift are the opposite shift in the encryption process.

(3) Inverse Matrix Multiplication by Constants. This stage is the inverse of a matrix multiplication by constants in the encryption process. Inverse multiplication of matrices with constant matrix is done in $\mathrm{GF}\left(2^{4}\right)$.

(4) Inverse Transpose. Inverse transpose is equal to the inverse transpose. To return to the original state matrix, transpose is performed again.

(5) Inverse Adds Round Key. This stage is the reverse of the process add a round key at the stage of encryption. Matrix elements will do the same xor operation as it exists at this stage but with the encryption subkey inverted schedule order. The process of getting a subkey of the master key is equal to the encryption stage.

Later on, the ciphertext goes through the five stages of decryption changing into plaintext and the resulting data can be used by the mobile payment application. The number of decryption stages is less than encryption stages because at the decryption there is no matrix pattern formation. After decryption is performed, the mobile payment application can retrieve the desired data on the location of the data stored in a matrix pattern.

3.3. DRE as a Token. The purpose DRE made was to secure the data exchanged during payment transactions using mobile applications. In mobile payment applications, in addition to serving as a DRE encryption algorithm that encrypts data, DRE also serves as a token. The token will ensure the transaction is valid from the start until the final stage of the transaction. With the token function, DRE is suitable for use in mobile payment applications that have an offline transaction mode because the offline transactions are highly susceptible to fraud (transactions with a prosthetic device/not registered), the transfer of the counterpart (man in the middle), or other attacks.

On a generated token there is information about the user's device, including IMEI and IMSI. In addition, there are also time information, user accounts, transaction data, and other information if needed. DRE rules used to generate tokens change on time. The rules are stored in a DRE database. In this research, a time frame utilized to bring forth the token is 
TABLE 5: Inverse substitution look-up table.

\begin{tabular}{|c|c|c|}
\hline & Input & Output \\
\hline \multirow{16}{*}{ Nibble 1} & 0000 & 1111 \\
\hline & 0001 & 0100 \\
\hline & 0010 & 0101 \\
\hline & 0011 & 1000 \\
\hline & 0100 & 0010 \\
\hline & 0101 & 1100 \\
\hline & 0110 & 1011 \\
\hline & 0111 & 0000 \\
\hline & 1000 & 1010 \\
\hline & 1001 & 1110 \\
\hline & 1010 & 1001 \\
\hline & 1011 & 0111 \\
\hline & 1100 & 1101 \\
\hline & 1101 & 0011 \\
\hline & 1110 & 0001 \\
\hline & 1111 & 0110 \\
\hline \multirow{16}{*}{ Nibble 2} & 0000 & 1110 \\
\hline & 0001 & 0111 \\
\hline & 0010 & 0100 \\
\hline & 0011 & 1011 \\
\hline & 0100 & 0101 \\
\hline & 0101 & 1001 \\
\hline & 0110 & 1111 \\
\hline & 0111 & 1000 \\
\hline & 1000 & 0011 \\
\hline & 1001 & 0110 \\
\hline & 1010 & 1101 \\
\hline & 1011 & 1010 \\
\hline & 1100 & 0010 \\
\hline & 1101 & 0000 \\
\hline & 1110 & 1100 \\
\hline & 1111 & 0001 \\
\hline
\end{tabular}

based along the order of the day in the year (day of the year). Figure 9 illustrates a block diagram of DRE as a token.

Security or authentication process performed by DRE on payment transactions using mobile applications is as follows.

(1) When a transaction starts, the two devices that will make transactions generate tokens.

(2) Tokens are then exchanged between the payer and the merchant (beneficiary paid). At the time of the exchange of data, in addition to the token sent, the device information including IMEI and IMSI is also sent unencrypted.

(3) Each device will check the given token by decrypting it using the existing rules on the DRE data base, in mobile payment applications. The merchant's token will be decrypted on the payer's device so that the

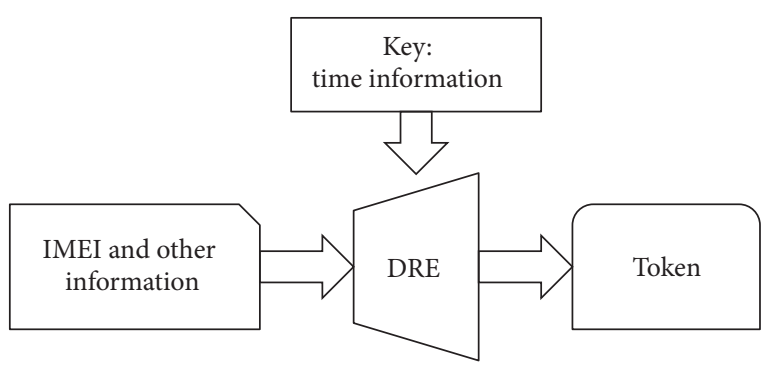

Figure 9: Token generator.

payer will acquire the IMEI and IMSI of the merchant's device, as well as other data. The payer's token will be decrypted on the merchant's device, so that the payer will acquire the IMEI and IMSI of the payer's device, as well as other data.

(4) IMEI and IMSI following the token decryption will be compared with the IMEI and IMSI sent in an unencrypted format (plaintext). If the IMEI and IMSI of the decrypted result are the same with IMEI and IMSI sent in plaintext format, so, the counterpart device is valid for transactions.

The token will always exchange during transmission of data between the two devices. Thus, fraud by third-party devices can be prevented when the transaction takes place.

\section{Implementation and Testing}

4.1. Implementation. Distance (Hamming distance) is numbers of different bits between two words (here is between two code words). The largest distance can be achieved between two code words is half of the total length of a code word.

Here, the distance metric is employed for making maximum differences between a plaintext and a ciphertext. The maximum difference is half of the length of the plaintext because $100 \%$ difference in binary means the same as the plaintext (converting all bits).

To produce ciphertext which has a distance approaching half the length of the plaintext, the election rules are required. This is also done so that the encryption and decryption process is working well. The rules are generated by the pattern generator. The pattern generator selects at random pattern by using a random function with various rules given. Here are the rules chosen randomly.

(1) Placement pattern input of the matrix

(2) The substituted matrix elements

(3) The rows and columns shifted and amounts of shift operation

(4) The constant matrix, and

(5) The master key to add a round key stage

Placement of input (plaintext) was randomly selected from the possibilities. There are no criteria for the selection of rules specifically for this stage. For cyclic shift, selected 
TABLE 6: Distance comparison between with and without random function.

\begin{tabular}{|c|c|c|}
\hline \multirow[b]{2}{*}{ Number } & \multicolumn{2}{|c|}{ Distance between input and output } \\
\hline & $\begin{array}{l}\text { Rule selection without } \\
\text { random function }\end{array}$ & $\begin{array}{l}\text { Rule selection with } \\
\text { random function }\end{array}$ \\
\hline 1 & 271 & 243 \\
\hline 2 & 265 & 252 \\
\hline 3 & 267 & 264 \\
\hline 4 & 267 & 236 \\
\hline 5 & 279 & 237 \\
\hline 6 & 280 & 254 \\
\hline 7 & 272 & 264 \\
\hline 8 & 270 & 264 \\
\hline 9 & 272 & 259 \\
\hline 10 & 273 & 261 \\
\hline Average & 271.6 & 253.4 \\
\hline
\end{tabular}

range scale is of $0-7$ with a random shift. Rows or columns shifted are selected randomly from all the possibilities. For matrix multiplication by a constant, the constant is selected from the variety of $1-15$. Constants may not be 0 to be decrypted. Constants also must not be worth more than 15 to be multiplied in $\mathrm{GF}\left(2^{4}\right)$.

The rule selection with and without random function will result in differences in distance between the input and output; the way this election may affect the performance of the DRE. Table 6 shows 10 data comparisons between the input and output as a result of the rule selection with and without the random function. The data used is 512 bits.

It is shown in Table 6 that by using a random function, the resulting distance will tend to close half of the length of the input, which will be close to 256 bits. This is because by using a random function, the rule option will be chosen evenly, or in other words, is not prone to certain rules option. With the prevalence of the rule option selected, the resulting combination of rules will have a good variety so that the resulting distance will be close to the best value.

Researches of Hamming distance computation on encryption have been done in [15-17]. Here, the DRE is employed in resulting maximum Hamming distance.

4.2. Distance Testing. DRE testing in this study is conducted by conducting five hundred times of testing. In each test, the data being input is different. One type of input data is the time stamp. Time stamp taken as input is a time stamp with a difference every thirty seconds. An accumulated time stamp is a time stamp accumulation every thirty seconds, while the nonaccumulated time stamp is a time stamp difference with thirty seconds earlier. Table 7 gives the results of DRE distance testing.

The input of DRE in these experiments is the data (code word) with a size of 512 bits (including salt), then the best distance between input and output code words is 256 bits. By appearing at the above test results, it can be concluded DRE has a proficient protection. In the entire operation or phase, DRE operation can produce an average distance between input and output code words of around 256 bits.

The distance testing between the output and other output code words with a slight difference in its input can also reflect the level of the strength of a cipher. If the distance among the outputs approaching half the length code word, then the results of encryption code word have different configuration of the better code word. In the entire operation, DRE can produce an average distance among the outputs of about 258 bits.

The operation or stage in the encryption process that provides most difference both in code word is the substitution operation. The substitution operation average distance is approximately 262 bits.

4.3. Time Testing. Time testing can represent the weight of a cipher algorithm run on a device or operating system. Here are the results of time testing for encryption and decryption. Tests are conducted as much as one thousand times using a Google Nexus S smartphone. A Sony Xperia SK17i smartphone is also used in this test which serves as a comparison. Table 8 is the result of time testing.

Judging from the results, it can be concluded that the DRE is not heavy or burdensome if run on mobile devices including smartphones. This is reinforced by the results of comparative testing using the device Sony Xperia SK17i. By using Sony Xperia SK17i, DRE encryption and decryption only takes about $18 \mathrm{~ms}$.

The test results are also compared with the time of Advanced Encryption Standard (AES) encryption [18]. Both tests are using the Google Nexus $S$ for one thousand times. Table 9 gives the results of comparative testing time between DRE and AES.

When compared with the speed of AES, DRE is much slower. By using the Google Nexus $S$, the time required to perform AES encryption is $0.62 \mathrm{~ms}$, while the time required to decrypt AES is $1.15 \mathrm{~ms}$. The cause DRE is lagging behind the AES in speed is due to the AES function being designed in the native $(\mathrm{C} / \mathrm{C}++)$ programming language, so it can perform faster in memory access and integer and floating-point calculations. Here, DRE process is designed and implemented using Android programming language [19, 20].

\section{Conclusion}

Dynamic Rule Encryption (DRE) algorithm is designed as a security element of a mobile payment application. In this paper, DRE serves to secure sensitive data as well as the authentication token when trading.

From the distance tests performed in this study, DRE has a good level of security because the average Hamming distance between the input and the output code words is about half of the length of the data being input. Thus, it will not be easy to trace the plaintext from the ciphertext. From the time of testing result, it is found that DRE is lightly used on mobile 
TABLE 7: Result of distance testing.

\begin{tabular}{lcc}
\hline Testing & Average of the distance to the input & Average of the distance to the previous output \\
\hline Using all stages (per 30 seconds) & 253.016 & 244.84 \\
Using all stages (add per 30 seconds & 253.016 & 257.55 \\
accumulation) & 262.064 & 175.73 \\
Substitution stage & 96.108 & 120.014 \\
The row cyclic sliding stage & 179.196 & 169.136 \\
The column cyclic sliding stage & 191.454 & 123.558 \\
Transpose stage & 164.148 & 143.326 \\
Multiplying stage in $G F\left(2^{4}\right)$ & 227.656 & 156.298 \\
Add round key stage & & \\
\hline
\end{tabular}

TABLE 8: Results of time testing.

\begin{tabular}{lcc}
\hline Device & $\begin{array}{c}\text { Time average of } \\
\text { encryption (ms) }\end{array}$ & $\begin{array}{c}\text { Time average of } \\
\text { decryption }(\mathrm{ms})\end{array}$ \\
\hline Google Nexus S & 19.537 & 23.519 \\
Xperia SK17 i & 18.262 & 18.428 \\
\hline
\end{tabular}

TABLE 9: Time average of DRE and AES.

\begin{tabular}{lcc}
\hline Cipher & $\begin{array}{c}\text { Time average of encryption } \\
(\mathrm{ms})\end{array}$ & $\begin{array}{c}\text { Time average of decryption } \\
(\mathrm{ms})\end{array}$ \\
\hline DRE & 19.537 & 23.519 \\
AES & 0.62 & 1.147 \\
\hline
\end{tabular}

devices because it has a fair execution time, not less than $24 \mathrm{~ms}$.

\section{Competing Interests}

The author declares that he has no competing interests.

\section{References}

[1] F. A. W. Wirawan and E. Oktivera, "Analysis on the implementation of digital marketing towards motorbike transport service case study: GO-JEK (online taxi motorbike) Jakarta, Indonesia," in Proceedings of the 2nd International Conference on Information Technology Systems and Innovation (ICITSI '15), pp. 1-6, Bandung-Bali, Indonesia, November 2015.

[2] S. Budi and H. T. Sukmana, "Developing mobile-based academic information system: a case study at Islamic State University (UIN) Syarif Hidayatullah Jakarta," in Proceedings of the 4th International Conference on Cyber and IT Service Management, Bandung, Indonesia, April 2016.

[3] E. Husni and S. Purwantoro, "Shopping application system with Near Field Communication (NFC) based on Android," in Proceedings of the International Conference on System Engineering and Technology (ICSET '12), pp. 1-6, IEEE, Bandung, Indonesia, September 2012.

[4] E. Husni and A. Ariono, "Development of integrated mobile money system using Near Field Communication (NFC): smartphone and electronic data capture," in Proceedings of the 8th International Conference on Telecommunication Systems Services and Applications (TSSA '14), pp. 1-6, IEEE, Bali, Indonesia, October 2014.

[5] E. Husni, Kuspriyanto, N. Basjaruddin, T. Purboyo, S. Purwantoro, and H. Ubaya, "Efficient tag-to-tag Near Field Communication (NFC) protocol for secure mobile payment," in Proceedings of the International Conference on Instrumentation, Communication, Information Technology and Biomedical Engineering (ICICI-BME '11), pp. 97-101, Bandung, Indonesia, November 2011.

[6] E. Husni, B. Leksono, and M. R. Rosa, "Digital signature for contract signing in service commerce," in Proceedings of the International Conference on Technology, Informatics, Management, Engineering and Environment (TIME-E '15), pp. 111-116, IEEE, Samosir, Indonesia, September 2015.

[7] A. J. Elbirt, W. Yip, B. Chetwynd, and C. Paar, "An FPGA-based performance evaluation of the AES block cipher candidate algorithm finalists," IEEE Transactions on Very Large Scale Integration (VLSI) Systems, vol. 9, no. 4, pp. 545-557, 2001.

[8] W. F. Ehrsam, S. M. Matyas, C. H. Meyer, and W. L. Tuchman, "A cryptographic key management scheme for implementing the Data Encryption Standard," IBM Systems Journal, vol. 17, no. 2, pp. 106-125, 1978.

[9] H. B. Kazemian and L. Meng, "Neuro-fuzzy control for MPEG video transmission over bluetooth," IEEE Transactions on Systems, Man and Cybernetics Part C: Applications and Reviews, vol. 36, no. 6, pp. 761-771, 2006.

[10] J. Daemen and V. Rijmen, "The first 10 years of advanced encryption," IEEE Security and Privacy, vol. 8, no. 6, pp. 72-74, 2010.

[11] Y. Tang and L. Zhang, "Adaptive bucket formation in encrypted databases," in Proceedings of the IEEE International Conference on e-Technology, e-Commerce and e-Service (EEE-05 '05), pp. 116-119, Hong Kong, China, March-April 2005.

[12] N. Ben Hadjy Youssef, W. El Hadj Youssef, M. Machhout, R. Tourki, and K. Torki, "Instruction set extensions of AES algorithms for 32-bit processors," in Proceedings of the 48th Annual IEEE International Carnahan Conference on Security Technology (ICCST '14), pp. 1-5, Rome, Italy, October 2014.

[13] Z. Guosheng and W. Jian, "Security analysis and enhanced design of a dynamic block cipher," China Communications, vol. 13, no. 1, pp. 150-160, 2016.

[14] A. J. Paul, P. Mythili, and J. K. Paulus, "Matrix based key generation to enhance key avalanche in advance encryption standard," in Proceedings of the International Conference on VLSI, Communication \& Instrumentation, pp. 31-34, Kottayam, India, April 2011. 
[15] A. Jarrous and B. Pinkas, "Secure computation of functionalities based on Hamming distance and its application to computing document similarity," International Journal of Applied Cryptography, vol. 3, no. 1, pp. 21-46, 2013.

[16] J. Bringer, H. Chabanne, and A. Patey, "SHADE: Secure HAmming DistancE computation from oblivious transfer," in Financial Cryptography and Data Security, vol. 7862 of Lecture Notes in Computer Science, pp. 164-176, Springer, Berlin, Germany, 2013.

[17] F. Guo, W. Susilo, and Y. Mu, "Distance-based encryption: how to embed fuzziness in biometric-based encryption," IEEE Transactions on Information Forensics and Security, vol. 11, no. 2, pp. 247-257, 2016.

[18] Lara-Niño, C. Andrés, M.-S. Miguel, and D.-P. Arturo, "An evaluation of AES and present ciphers for lightweight cryptography on smartphones," in Proceedings of the 26th International Conference on Electronics, Communications and Computers (CONIELECOMP '16), pp. 87-93, Cholula, Mexico, February 2016.

[19] J. K. Lee and J. Y. Lee, "Android programming techniques for improving performance," in Proceedings of the 3rd International Conference on Awareness Science and Technology (iCAST '11), pp. 386-389, IEEE, Dalian, China, September 2011.

[20] Y. Kwon, S. Lee, H. Yi et al., "Mantis: efficient predictions of execution time, energy usage, memory usage and network usage on smart mobile devices," IEEE Transactions on Mobile Computing, vol. 14, no. 10, pp. 2059-2072, 2015. 


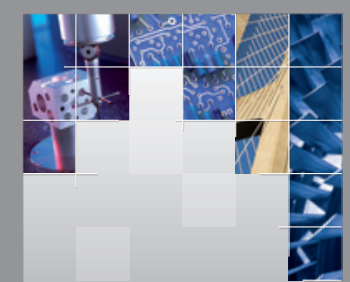

\section{Enfincering}
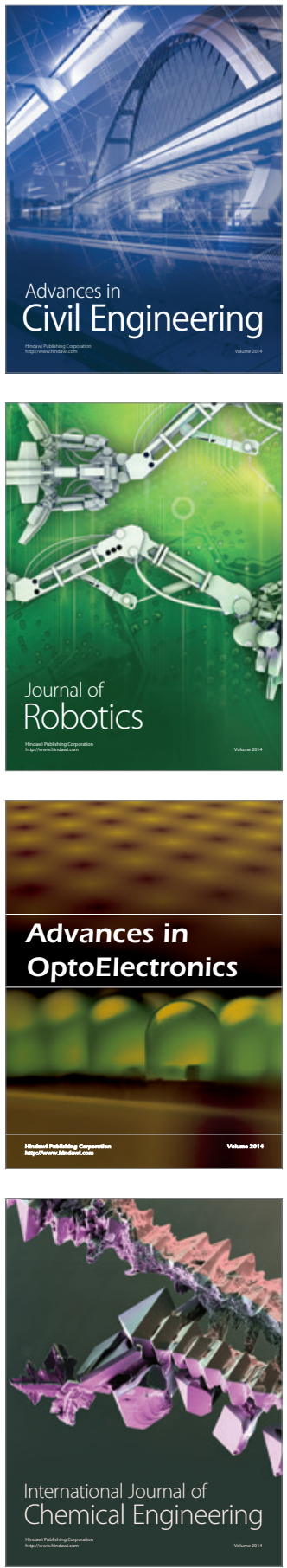

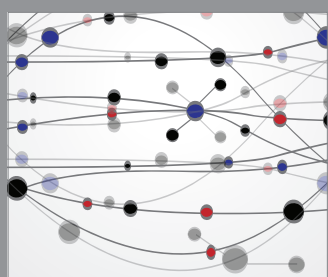

The Scientific World Journal

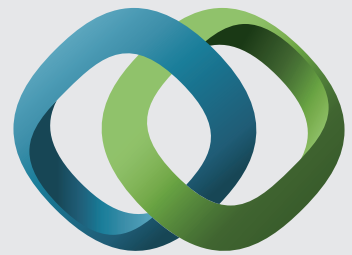

\section{Hindawi}

Submit your manuscripts at

https://www.hindawi.com
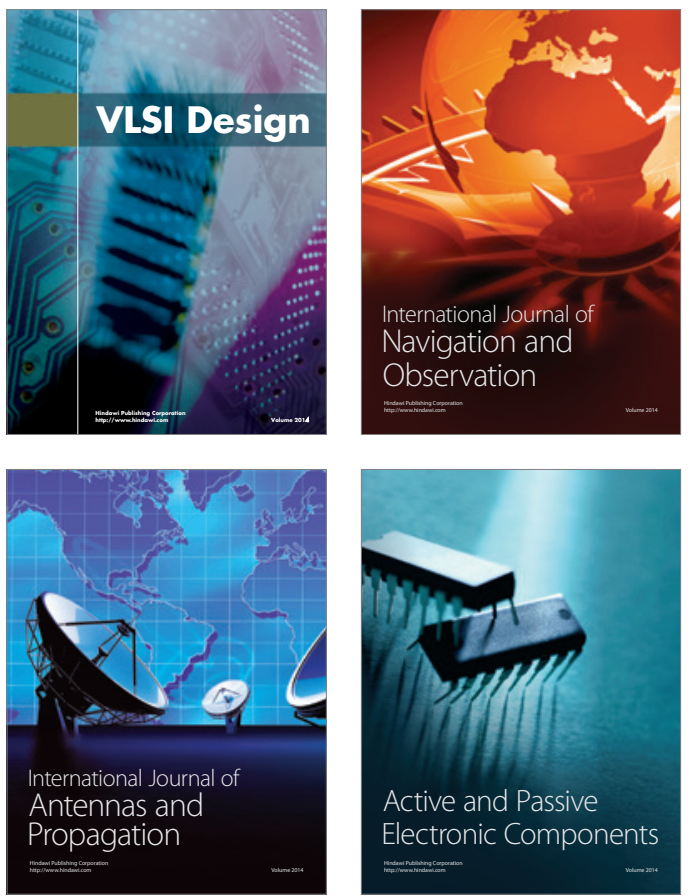
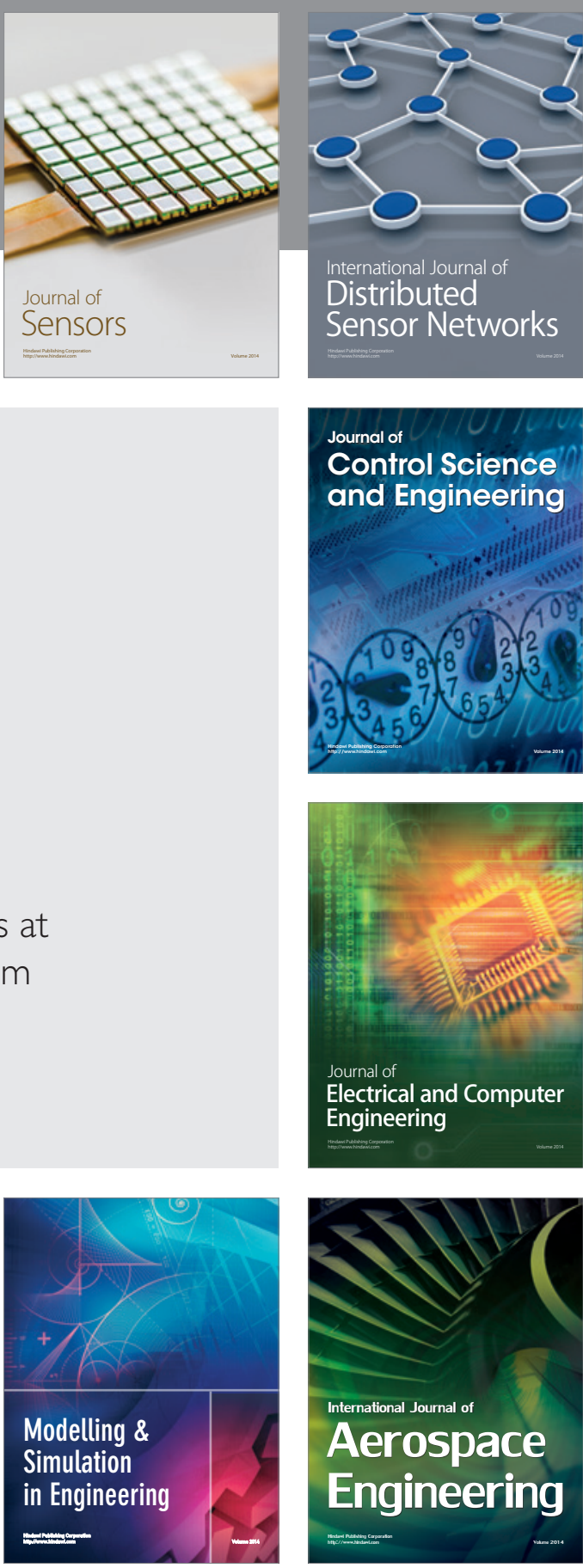

International Journal of

Distributed

Sensor Networks

$-$

Joumal of

Control Science

and Engineering
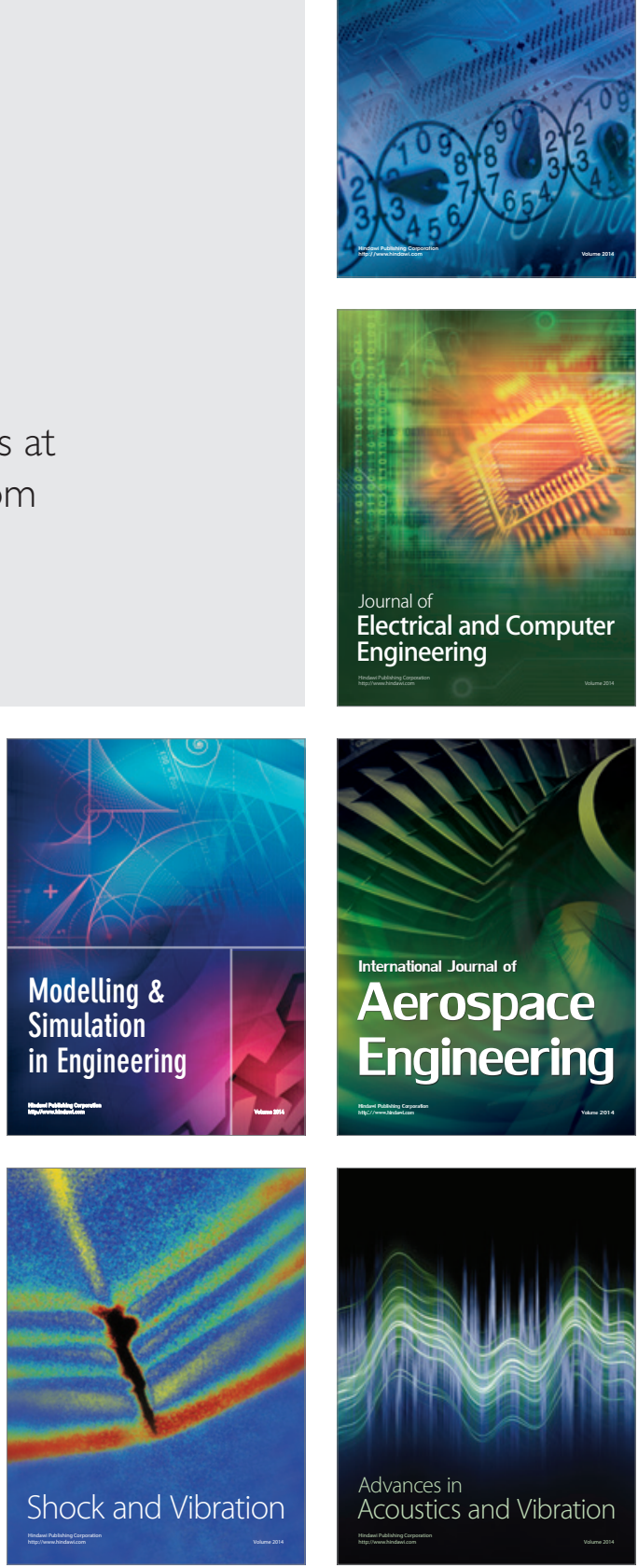